\title{
The Many-Property Problem is Your PRoblem, ToO
}

\author{
JUSTIN D'AMBROSIO \\ Australian National University
}

\begin{abstract}
The many-property problem has traditionally been taken to show that the adverbial theory of perception is untenable. This paper first shows that several widely accepted views concerning the nature of perception-including both representational and nonrepresentational views-likewise face the many-property problem. It then presents a solution to the many-property problem for these views, but goes on to show how this solution can be adapted to provide a novel, fully compositional solution to the manyproperty problem for adverbialism. Thus, with respect to the many-property problem, adverbialism and several widely accepted views in the philosophy of perception are on a par, and the problem is solved.
\end{abstract}

\section{Introduction}

The many-property problem was originally posed as a problem for the adverbial theory of perception, and is often taken to show that the adverbial theory is untenable. Until now, philosophers of perception have taken the problem to be unique to adverbialism. This paper shows that this assumption is mistaken: the problem afflicts any view of the nature of perception-representationalist or otherwise-on which perceptual experience involves a subject bearing a relation to an uninstantiated property or complex of properties. ${ }^{1}$

Luckily for such views, I provide a general solution to the many-property problem that makes use of a standard, widely-used semantic mechanism. However, this solution is one that can be easily adapted to yield a novel, fully compositional solution to the many-property problem for adverbialism. Further, this

Contact: Justin D'Ambrosio <justin.d'ambrosio@anu.edu.au>

${ }^{1}$ As we will see below, this category includes (i) all property representationalist views, (ii) relational views of perception on which we can be perceptually aware of uninstantiated properties, and (iii) views that treat the contents of perception as gappy. 
solution is superior to others in the literature in that it avoids objections to which such proposals are subject, and provides a complete explanation of the systematic entailments that hold between adverbially modified states of sensing. Thus, with respect to the many-property problem, adverbialism and a wide range of standard views in the philosophy of perception are on a par: all of them face, and can solve, the many-property problem.

\section{The Many-Property Problem}

The adverbial theory of perception was originally developed to provide an alternative to the act-object conception of experience: the view that in every perceptual experience, there is an act of awareness and an object of which we are aware. Adverbial theories attempt to provide an alternative to this view by arguing that the structure of perception is not fundamentally relational-for the adverbialist, sensory awareness does not consist in a relation to an object-and they articulate this view of the structure of perception by paraphrasing the complements of direct-object perceptual reports adverbially. ${ }^{2,3}$ The basic adverbialist proposal is that, at least in cases of hallucination, if Mary senses something green, there is not some object which she senses that is green. Rather, Mary senses in a certain way: greenly.

The many-property problem is a dilemma forced on the adverbialist when she tries to give an account of the situation described by the following ascription:

(1) Mary senses a green square and a red circle. ${ }^{4}$

${ }^{2}$ Adverbialism about perception was originally defended by Ducasse 1942 and Chisholm [1956], and then later by Sellars 1975] and Tye [1975, 1984. For many years, however, most philosophers of perception treated adverbialism as untenable on its own terms, and thought that its benefits were available to other views-typically representationalist views. Recently, however, there has been some renewed discussion of adverbial views of perception and intentionality, for instance, by Kriegel [2007, 2008, 2011], Mendelovici [2018, Ch. 9], Banick [forthcoming], Bourget |forthcoming|, and D'Ambrosio |2019|.

${ }^{3}$ Adverbialism as a theory of perception is importantly distinct from adverbialism concerning other aspects of our mental lives, including qualia, the emotions, and attention. The form of adverbialism with which I am concerned is a view concerning the fundamental nature of perception.

${ }^{4}$ In what follows, I will uniformly use the verb "senses" in my examples, which I take to express a state of sensory awareness that is present in every instance of perceptual experience. I make this choice because (a) "senses" is the verb used in traditional debates over the manyproperty problem, (b) most views to which my arguments apply hold that we can sense, or 
The adverbialist might paraphrase (1) as follows:

(2) Mary senses greenly and squarely and redly and circularly.

But this paraphrase fails to distinguish Mary's sensing from one that involves the same properties, but pairs them differently; it fails to distinguish (1) from (3):

(3) Mary senses a red square and a green circle.

Call this the expressive problem. In order to address this problem, the adverbialist often tries to form complex adverbs, traditionally by paraphrasing (1) in ways such as the following:

(4) Mary senses (green-and-square)-ly and (red-and-circular)-ly. ${ }^{5}$

However, the formation of complex adverbs in this way undermines the status of "green" and "square" and "red" and "circular" as genuine syntactic constituents of the sentence, and so appears to undermine the adverbialist's ability to infer from (3) to its obvious consequences such as (5) and (6):

(5) Mary senses a square.

(6) Mary senses something green.

Call this the inferential problem. The many-property problem is the dilemma formed from the expressive problem and the inferential problem. ${ }^{6}$

be sensorily aware of, uninstantiated properties, and (c) it meshes well with the view, due to Johnston, that I will use below to illustrate the broader category of views in which I am interested. However, I just as well could have made use of "perceives", "is aware of", "is perceptually aware of", or "is sensorily aware of" to formulate my examples, so long as none of these verbs require the existence of particulars instantiating the relevant properties.

5 On this traditional approach to forming complex adverbs, which is roughly the view given in Tye 1975l, "(green-and-square)-ly" and (red-and-circular)-ly" are syntactic primitives. This is not a particularly plausible approach to the semantics of adverbial modification, since it is not compositional. Below, in solving the many-property problem, I will present a compositional way of forming such adverbs, but for the moment, in presenting the problem, I will make use of the traditional paraphrases.

${ }^{6}$ The many-property problem was originally proposed by Frank Jackson [1975]. There have been numerous attempts to respond to it, including by Tye [1975, 1984, 1989], Sellars [1975], and more recently by Kriegel [2007, 2008]. These attempts have met with resistance from Woodling [2016] and Grzankowski [2018], and have even occasioned a strengthening of the original problem due to Dinges [2015]. 


\section{Uninstantiated Properties in Perception}

We have seen that adverbialism faces the many-property problem, but in fact, many other theories do too-namely, those that posit that perceptual experience involves a relation between a subject and an uninstantiated property. To see this, it will be helpful to consider a representative view: that of Mark Johnston [2004].$^{7}$ Johnston holds that the objects of hallucination are sensible profiles, where sensible profiles are complex properties (or: complexes of properties). ${ }^{8}$ To use Johnston's example, in the case where I hallucinate an Italian greyhound under a desk, I am related to a complex consisting of the property of being an Italian greyhound, the property of being a desk, and perhaps the relation of $x$ being under $y .{ }^{9}$ Such sensible profiles, on Johnston's view, serve as the objects of sensory awareness whether or not we are hallucinating. When we are hallucinating, such properties will be uninstantiated or partially uninstantiated. When we perceive veridically, such profiles will be instantiated by collections of concrete particulars that have the properties and stand in the relations the sensible profile comprises.

Now consider the following perceptual ascription:

Mary senses a green square and a red circle.

On Johnston's view, the object of Mary's awareness is a complex property-a sensible profile-whose components include the properties of being green, being

7I choose Johnston's view here for definiteness and clarity, but my arguments apply to any view of perception that involves the perceiving subject bearing a relation to an uninstantiated property, whether or not the relation is one of awareness, and whether or not the relation is representational. Such views are numerous. For instance, the view that in hallucinatory perceptual experience, we are aware of uninstantiated properties is held by Bealer [1982], Dretske [1999] 2003], Foster [2000], Forrest 2005], Johnston [2004], and Tye 2014a b], among others, although Johnston is not a representationalist. The view that in perception, we bear a relation that is not an awareness relation to uninstantiated properties is defended by Pautz 2007. The arguments also apply to the view that the contents of hallucination are gappy, defended by Tye 2009] and Schellenberg [2013, 2014], among others.

${ }^{8}$ Johnston moves between these two different phrases. As we will see presently, this equivocation matters.

${ }^{9}$ It is important to note that sensible profiles can include uninstantiated relations, such as the relation of being under. However, insofar as views such as Johnston's allow uninstantiated relations to figure into the complex properties that we sense, such views also face the so-called "many-relations" problem, posed by Dinges 2015. As we will see, the solution I provide below solves both the many-property and many-relations problems for both property theories and adverbialism. 
square, being red, and being a circle, and in (7), "senses" expresses a relation to that property. In order to serve as the semantic value of the complement of the verb, the complex property must be derived from the ordinary semantic value of "a green square and a red circle" via a type-shift. ${ }^{10}$

So far so good. But what distinguishes a sensible profile of a green square and a red circle from a sensible profile of a green circle and a red square? How can we distinguish (7) from (8)?

(8) Mary senses a green circle and a red square.

In a hallucinatory case, there are no particulars available to instantiate the two pairs of properties, and so something else must distinguish perceptual situations in which they are paired differently. What can achieve such differentiation? Johnston's intention is clearly for sensible profiles to be complex properties: properties that are built up from other properties in a way that mimics patterns of co-instantiation. Thus, the property that Mary is aware of in the hallucinatory case must have structure: Mary must be perceive the property of being (greenand-square) and (red-and-circular).

The standard way of forming such properties is to make use of an abstraction operation such as lambda abstraction. Lambda abstraction allows us to construct complex properties that mimic patterns of coinstantiation as follows:

(9) $\lambda x \lambda y[\operatorname{green}(x) \wedge \operatorname{square}(x) \wedge \operatorname{red}(y) \wedge \operatorname{circular}(y)]$

(10) $\quad \lambda x \lambda y[\operatorname{green}(x) \wedge \operatorname{circular}(x) \wedge \operatorname{red}(y) \wedge$ square $(y)]$

In (9), the bound variables pair the properties of being green and square, and the properties of being red and circular. The logical forms of (7) and (8) are then given by (11) and (12), respectively:

(11) $\quad \operatorname{sense}(\operatorname{Mary}, \lambda x \lambda y[\operatorname{green}(x) \wedge \operatorname{square}(x) \wedge \operatorname{red}(y) \wedge \operatorname{circular}(y)])$

\footnotetext{
${ }^{10} \mathrm{~A}$ type shift is a change in the type of semantic value that a semantic theory assigns to a particular expression, typically to correct for a mismatch between the argument-type accepted by a verb's denotation and the type of semantic value assigned to its argument. The ordinary semantic value of a complex noun phrase such as "a green square and a red circle" is a generalized quantifier. In a standard Montagovian system, such a quantifier is intensional, and of type $\langle s,\langle\langle s,\langle e, t\rangle\rangle, t\rangle\rangle$. In extensional theories, generalized quantifiers are of type $\langle\langle e, t\rangle, t\rangle$. We will see below how to shift an object of this type to the type of a property. See Hendriks 1993 for a systematic approach to type-shifting.
} 


$$
\text { sense(Mary, } \lambda x \lambda y[\operatorname{green}(x) \wedge \operatorname{circular}(x) \wedge \operatorname{red}(y) \wedge \operatorname{square}(y)])
$$

Since the properties to which Mary is related in (12) and (11) are distinct, it may appear that lambda abstraction allows Johnston's view to distinguish between (7) and (8), and so solve the many-property problem.

But drawing this conclusion would be a mistake. Johnston's view still confronts the inferential problem; his view does not yet have an explanation of why (7) should entail (13) or (14):

(13) Mary senses a green square.

(14) Mary senses a square.

The reason that Johnston's view still faces the inferential problem is that structural relations between properties-like the one between the properties denoted by "a green square" and "a square" - are not, on their own, sufficient to guarantee that relations of entailment hold between states of sensing those properties. Just because one senses $\mathrm{P}$, and $\mathrm{P}$ bears a structural relation to $\mathrm{Q}$, such as having $\mathrm{Q}$ as a component, it does not-absent further argumentation-follow that one senses $Q .{ }^{11}$

We can see this by noting that that while "senses" validates inferences such as the one from (13) to (14), and so "distributes" to any subproperty, many other verbs whose complements denote properties do not. Consider the following pair:

(15) Mary lacks a comfortable pillow.

(16) Mary lacks a pillow.

The inference from (15) to (16) is not valid-Mary can lack a comfortable pillow without lacking a pillow, despite the fact that "comfortable" serves as a constituent of "comfortable pillow" in the same way that "green" serves as a constituent of "green square". Moreover, (13) and (15) plausibly have the same logical form; both ascriptions involve intensional transitive verbs whose comple-

\footnotetext{
${ }^{11}$ One way of bringing this out is to notice that despite the fact that (11) and (12) have structured properties as arguments, their logical form, at least in first-order logic, is still that of a simple binary relation; they both have the form $\mathrm{V}($ Mary, $\mathrm{P})$. In order to determine which inferences such sentences underwrite, we need principles that go beyond those in first-order logic: we need principles that tell us how structural relations between properties determine relations between states that relate us to those properties.
} 
ments denote properties-i.e. both have the form given in (17):

$$
V[\text { Mary, } \lambda x[P(x) \wedge Q(x)]] .
$$

But while (13) and (15) have the same logical form, they have different entailments. Thus, the structural relations between the properties to which a subject bears relations are not sufficient to determine entailment relations between states in which those properties figure.

To take another example, imagine that John is playing a concerto. Then consider the contrast between (18) and (19):

a. John promised a long cadenza.

b. John promised a cadenza.

(19) a. John omitted a long cadenza.

b. John omitted a cadenza.

Clearly, (18-a) entails (18-b), but (19-a) does not entail (19-b), even though (18-a) has the same logical form as (19-a), and (18-b) has the same logical form as $(19-b) .{ }^{12}$ In fact, given that (18-a) and (19-b) are a minimal pair-they differ only in the verbs that they contain-the differences in entailment must be traceable to the differing lexical meanings of those verbs. Thus, in order to validate inferences between reports of states of sensing, it is not sufficient to point out that one property is a component of another, or that one property figures in the compositional derivation of a larger property-denoting expression. Rather, what is needed is an additional, verb-specific rule that specifies how relations between properties determine inferential relations between states of sensing those properties. What is required is to specify the monotonicity profile of the verb in question. This is the core of the inferential problem. ${ }^{13}$

\footnotetext{
12"Omits" is not the only verb for which such inference patterns fail: they also fail for verbs of resemblance, such as "resemble", "imitate", and "simulate", and for verbs such as "lack". It is a substantive question which intensional verbs validate adjective-drop inferences within their complements.

${ }^{13}$ This argument begins to show, contra Bourget |2017], that inferences within the complements of intensional ascriptions cannot be purely formal. Which inferences hold within the complement of an intensional verb will depend on the lexical meaning of the verb in question. I will discuss this point further below. However, I agree with Bourget that inferences involving so-called "special quantifiers", such as from "John wants a big dog" to "John wants something" are formal. This may well be due to the distinctive semantic features of "something", which are
} 
The result is that views in the philosophy of perception that posit relations to uninstantiated properties are crucially underspecified in exactly the way that adverbialism is underspecified; both can easily handle the expressive horn of the dilemma, but have nothing to say when they encounter the inferential horn. In order to address the inferential problem, Johnston must first make use of the strategy employing lambda abstraction above, and second, must give a rule specifying how the structural relations between properties determine relations of entailment between states of sensing those properties. In short, Johnston and the property-representationalist need a logic of sensible profiles: they need a logic of property perception. But such a logic is exactly what the adverbialist needs as well; they need a logic of adverbial modification.

Further, Johnston's view is merely illustrative. Insofar as many views hold that perceptual experience involves or consists in a relation to an uninstantiated property-at least in the hallucinatory case-this problem is very general. Arguments like the ones above can be marshalled against any theory of perception on which subjects can bear perceptual relations to uninstantiated properties, and these arguments hold whatever one's views on the nature of that relation. We can call such views property views. Perhaps the most common property view is property representationalism. Like Johnston, property representationalists typically hold that perceptual experience consists in awareness of a complex property, and hold that when perceptual experience is veridical, that property will be instantiated. However, unlike Johnston, they often claim, as Tye 2014a does, that the relation the subject bears to the property is one of "predicative representation". Notable defenders of such views are Dretske [1995, 1999, 2003] and Tye [2014a b], although there are many others as well.

Another form of the property view, defended by Pautz [2007, 2010], holds that in hallucination, we bear a theoretically defined relation to a complex uninstantiated property, but this relation is not one of awareness. My arguments apply to these views also-nothing about the arguments above is specific to views on which the relation we bear to an uninstantiated property is one of awareness. Like many property representationalists, Pautz recognizes the need to solve the expressive problem [see Pautz, 2007, pp. 498-499], but likewise fails to address the inferential problem.

outlined by Sainsbury 2018, Ch. 2]. Sainsbury argues that "something" is substitutional. 
Finally, the arguments above apply to the view, defended by Susanna Schellenberg [2013, 2014], that the content of a hallucinatory perceptual experience is "gappy". Schellenberg takes gappy contents to be modes of presentation that are associated with certain perceptual capacities. Schellenberg's gappy contents are, in many ways, like properties, albeit very finely individuated ones. But in the case where a subject hallucinates something with multiple properties-for instance, a green square-something must fuse the gappy contents together to account for the features of the unified experience. Once this fusion is accomplished, Schellenberg's view will likewise face the inferential problem. While I acknowledge that there are key differences between Schellenberg and standard property theorists, I will treat "property theorist" as a label applying to her as well. ${ }^{14}$

The feature that these views share, which makes them subject to the manyproperty problem, is that in cases of hallucination, they require formation of complex properties (or in Schellenberg's case, complex gappy contents) that mimic patterns of coinstantiation. But once they construct these properties, these views face the inferential problem, and are silent about how to solve it. ${ }^{15}$

\section{The Solution: Monotonicity}

The solution to the many-property problem, for both the property theorist and the adverbialist, is to treat the inferential problem as a problem concerning monotonicity inferences. Monotonicity inferences are ubiquitous in semantics, but most importantly for our purposes, they arise in the context of giving a semantics for intensional transitive verbs such as "seeks". ${ }^{16}$

\footnotetext{
${ }^{14}$ See Schellenberg [2013 p. 304] for a clear statement of the view that illustrates the need for a solution to the many-property problem.

${ }^{15}$ In what follows, I will continue to use Johnston's view to illustrate my points, while noting that my arguments generalize to all property views.

${ }^{16}$ Perhaps most basically, monotonicity inferences are the result of quantification; each quantifier has a so-called "monotonicity profile": a distinctive collection of monotonicity inferences that it validates. See van Benthem $[1984$ for discussion. Propositional attitude verbs underwrite monotonicity inferences because on the standard semantics for such verbs, given by Hintikka [1962], propositional attitudes involve quantification over worlds: propositional attitude verbs are modals. Thus, it is common for semanticists to argue over the monotonicity profiles of intensional verbs. See Forbes 2006], Zimmermann [2006], von Fintel and Heim [Unpublished, and Rubinstein 2017 for discussion.
} 
Monotonicity inferences typically arise in the complements of intensional verbs. In the case of intensional transitive verbs, these inferences arise in the direct object position, as we can see by noting that (20) implies (21):

(20) John seeks a book by Quine.

(21) John seeks a book.

Even when we interpret both (20) and (21) nonspecifically, so that John does not seek any particular book, the inference from (20) to (21) is valid. More generally, "seeks" validates the inference patterns in (22) and (23), where F 5 iff the extension of $\mathrm{F}$ is a subset of the extension of $\mathrm{G}$ :
a. $\quad \mathrm{S}$ seeks an $\mathrm{F}$
b. S seeks a G

where $\mathrm{F} \sqsubseteq \mathrm{G}$,

\section{a. S seeks an $\mathrm{F}$ and a $\mathrm{G}$}

b. S seeks an F,

When a verbal argument position validates inferences of these forms, the verb is monotonic upward in that position. ${ }^{17}$

The standard semantic explanation for this monotonicity behavior is that "seeks" has a covert modal element that quantifies over worlds in which the search is successful. ${ }^{18}$ One way of spelling out the behavior of this modal is the Quine-Hintikka Analysis of intensional verbs; it is this analysis that validates upward monotonicity. ${ }^{19}$ We can illustrate the Quine-Hintikka analysis informally using our example from above. On the Quine-Hintikka analysis, to seek a book

\footnotetext{
${ }^{17}$ By considering only these two inference patterns, I am simplifiying the monotonicity problem significantly. The problem for intensional transitive verbs becomes much more complicated once disjunctive and quantified complements are introduced. However, this simplification is justified because these two patterns are the ones relevant to the case of perception, and the ones that form the basis of the traditional inferential problem for adverbialism. For further discussion of the monotonicity profile of intensional verbs, see Forbes [2006] and Sainsbury 2018.

${ }^{18}$ Other intensional transitive verbs specify conditions appropriate to the kinds of activities they denote. For instance, desires may have satisfaction-conditions, debts may have discharge conditions, fears may have realization conditions, etc.

${ }^{19}$ The analysis is called the "Quine-Hintikka Analysis" because it has its roots in Hintikka's semantics for propositional attitude verbs, which validates upward monotonicity for propositional attitudes. Quine then proposed to decompose non-propositional attitude verbs into propositional attitude verbs in a way that would make them susceptible to Hintikka's analysis.
} 
by Quine is to be the agent of a search such that, in every world in which one's search is successful, one finds a book by Quine. Put schematically, a subject $S$ seeks NP iff in every world where the search is successful, the subject $S$ finds NP. Monotonicity inferences are then validated by considering the systematic relations that hold between the success-conditions of different searches. ${ }^{20}$

One well-known implementation of the Quine-Hintikka analysis is given by Zimmermann [2006. Zimmermann treats "seeks" as a relation between an agent and a property, and then uses the Quine-Hintikka analysis to secure upward monotonicity. This implementation has two steps: first, Zimmermann gives a compositional semantics that treats intensional transitive verbs as denoting relations between an agent and a property. Zimmermann then applies the QuineHintikka analysis to show how the property specifies the success-conditions for the event, and validates upward monotonicity. I discuss these two steps in turn.

In the compositional component, Zimmermann [2006, p. 736] first shows how to take the the ordinary semantic value of a noun phrase such as "a book by Quine"-which is of generalized quantifier type-and convert it to the type of a property. He does this by employing a type-shifting principle that he calls Existential Lowering:

(24) Existential Lowering: From $\lambda P[Q(P)]$ of type $\langle\langle e, t\rangle, t\rangle$ to $(\lambda x[R(x)]$ of type $\langle e, t\rangle$, where $R$ is the restrictor of $Q$. Zimmermann 2006, p. 736, notation modified $]^{21}$

This type shift takes the ordinary semantic value of a quantificational nounphrase, a generalized quantifier, and returns the semantic value of its restrictor, which is a property. Zimmermann then employs this type shift in the compositional semantics for sentences such as (25):

(25) John seeks a book by Quine.

\footnotetext{
${ }^{20}$ This is analogous to inferences that arise in the complements of propositional attitude verbs, except that in the propositional case, instead of success-worlds, we considering worlds at which the proposition in question is true.

${ }^{21}$ For example, if $Q$ is the quantifier denoted by "some $\operatorname{dog}$ ", $\lambda P \exists x[\operatorname{dog}(x) \wedge P(x)]$, the type shift yields the property that serves as $Q^{\prime}$ s restrictor, $\lambda x[\operatorname{dog}(x)]$.
} 
(26)

John seeks a book by Quine $\operatorname{seek}^{\prime}\left(\operatorname{John}^{\prime}, \lambda x\left[\operatorname{book}^{\prime}(x) \wedge\right.\right.$ by-quine $\left.\left.(x)\right]\right)$

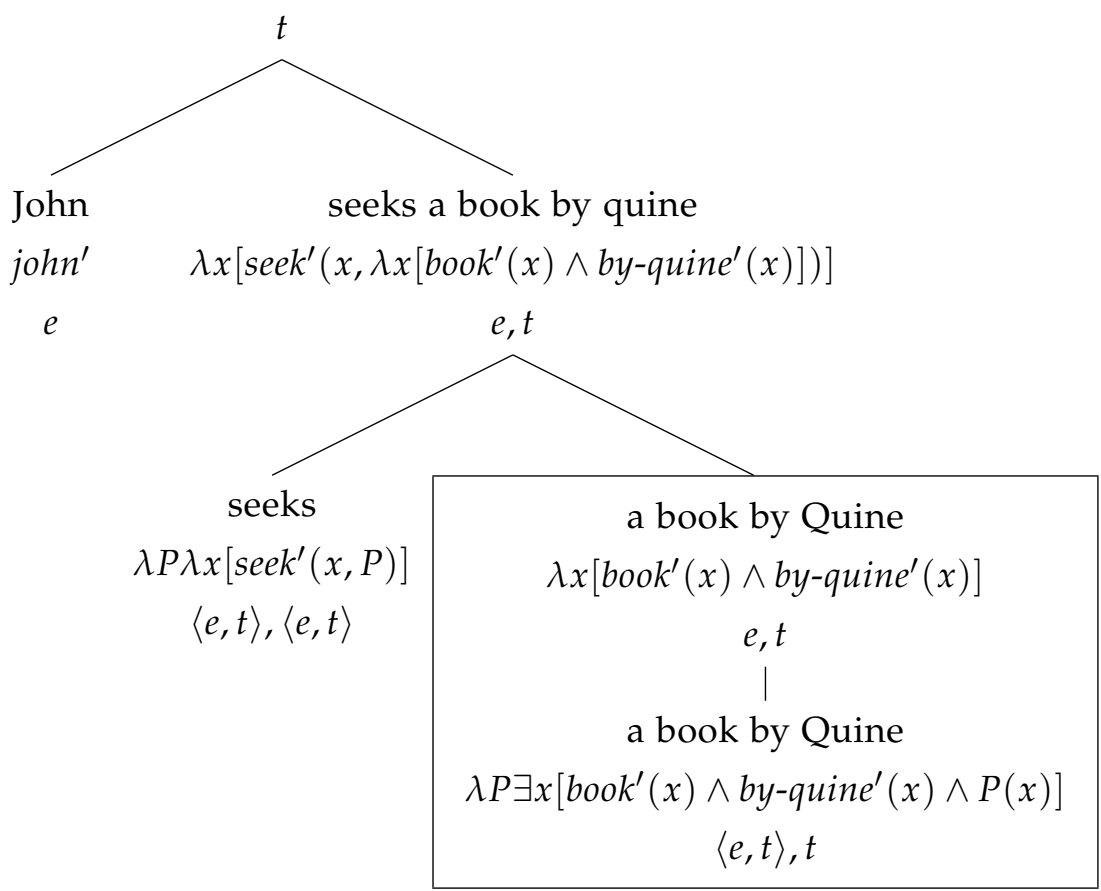

The principle of Existential Lowering is applied in the boxed portion of the derivation. The principle can be seen as being triggered by a type-mismatch between the verb and the ordinary semantic value of the quantificational noun phrase. The principle shifts the type of the argument, and so corrects the mismatch. The result of the derivation is that (25) says that John stands in the seekrelation to a complex property-the property of being a book by Quine.

But this derivation is not yet sufficient to secure upward monotonicity; validating upward-monotonic inferences requires applying the Quine-Hintikka analysis. Zimmermann provides this analysis as follows:

(27) Quine + Hintikka Analysis of "seek" (with events)

For any property $P$, individual $x$, world $w$, and time $t$ the following holds: $\operatorname{seek}(P)(x)(w)(t)=1$ iff there is a search act $e$ performed by $x$ in $w$ at $t$ such that the property of being found by $x$ overlaps the extension of $P$ at $\left(w^{\prime}, t^{\prime}\right)$, for any worlds $w^{\prime}$ and times $t^{\prime}$ such that $(w, t) T\left(w^{\prime}, t^{\prime}\right)$. Zimmermann [2006, p. 744] 
Above, the relation $T$ is an accessibility relation that holds between world/time pairs just in case the event that takes place at one world and time is successful at another, and $P$ is a possibly complex property. Thus, the analysis of "seek" formalizes the idea that in any worlds in which the act of searching is successful, $x$ finds an instance of $P$.

The feature of the Quine + Hintikka analysis that allows it to validate upward monotonicity is the quantification over worlds and times in the last clause of the analysis. ${ }^{22}$ Given this quantification, we can give the following argument to show that upward monotonicity is valid. Suppose, without loss of generality, that John seeks a book by Quine. Any world in which this search is successful is one in which he finds a book by Quine. But any world in which he finds a book by Quine is one in which he finds a book. Therefore, in every world in which John's search is successful, he finds a book. But by the right-to-left direction of the biconditional above, it follows that John seeks a book. So the definition validates inferences such as the one in (22). Similar reasoning yields that (27) validates the inference in (23), except that in this case the properties in question will be conjunctive properties. ${ }^{23}$

\section{Solving the Problem for Property Views}

The tools used to address the monotonicity inferences that hold in the complements of intensional transitive verbs, illustrated with the case of "seeks" above, can be used to solve the many-property problem for the property theorist. Since Zimmermann treats "seeks" as a relation to a property, with minor modifications, his analysis can be converted into an analysis of the verb that expresses the relation of sensory awareness to an uninstantiated sensible profile. To see this consider our example from above:

(28) Mary senses a green square and a red circle.

First, we need to provide a compositional semantics on which (28) has the logical

\footnotetext{
${ }^{22}$ In fact, Hintikka's original analysis secured upward monotonicity for all propositional attitude verbs, which leads to the problem of logical omniscience.

${ }^{23}$ On the view proposed by Forbes [2006], validating inferences involving conjunctive NPs in the complements of intensional verbs requires stating an additional postulate governing NPs of this form. I omit this complication here, but refer the reader to Forbes 2006, p. 99].
} 
form of a relation between Mary and a complex property. A slight generalization of Existential Lowering allows us to do just this. The original version of Existential Lowering only applied to simple properties. But a perfectly analogous principle allows us to type-shift the denotations of complex quantificational expressions, such as "a green square and a red circle", to the type of complex properties. $^{24}$ The generalized form of Existential Lowering takes the ordinary semantic value of a quantified NP and returns the denotation of its restrictor, as in (29):

(29)

$$
\begin{aligned}
& \lambda P \exists x \exists y\left[\operatorname{green}^{\prime}(x) \wedge \operatorname{square}^{\prime}(x) \wedge \operatorname{red}^{\prime}(y) \wedge \operatorname{circle}^{\prime}(y) \wedge P(x) \wedge P(y)\right] \text { of type } \\
& \langle\langle e, t\rangle, t\rangle \text { to } \lambda x \lambda y\left[\operatorname{green}^{\prime}(x) \wedge \operatorname{square}^{\prime}(x) \wedge \operatorname{red}^{\prime}(y) \wedge \operatorname{circle}^{\prime}(y)\right] \text { of type }\langle e,\langle e, t\rangle\rangle
\end{aligned}
$$

We can then deploy this type-shift in the boxed portion of the derivation in (30), where $Q$ stands in for $\lambda P \exists x \exists y\left[\operatorname{green}^{\prime}(x) \operatorname{square}^{\prime}(x) \wedge \operatorname{red}^{\prime}(y) \wedge \operatorname{circle}^{\prime}(y) \wedge P(x) \wedge\right.$ $P(y)]$ :

${ }^{24}$ The only difference here is that $\lambda x \lambda y\left[\operatorname{green}^{\prime}(x) \wedge \operatorname{square}^{\prime}(x) \wedge \operatorname{red}^{\prime}(y) \wedge \operatorname{circle}^{\prime}(y)\right.$ is of type $\langle e,\langle e, t\rangle\rangle$, as opposed to type $\langle e, t\rangle$. This is just a feature of the kind of properties that hold of two distinct objects. 
(30) Mary senses a green square and a red circle $\operatorname{sense}^{\prime}\left(\operatorname{Mary}^{\prime}, \lambda x\left[\operatorname{green}^{\prime}(x) \wedge \operatorname{square}^{\prime}(x) \wedge \operatorname{red}^{\prime}(y) \wedge \operatorname{circle}^{\prime}(y)\right]\right)$

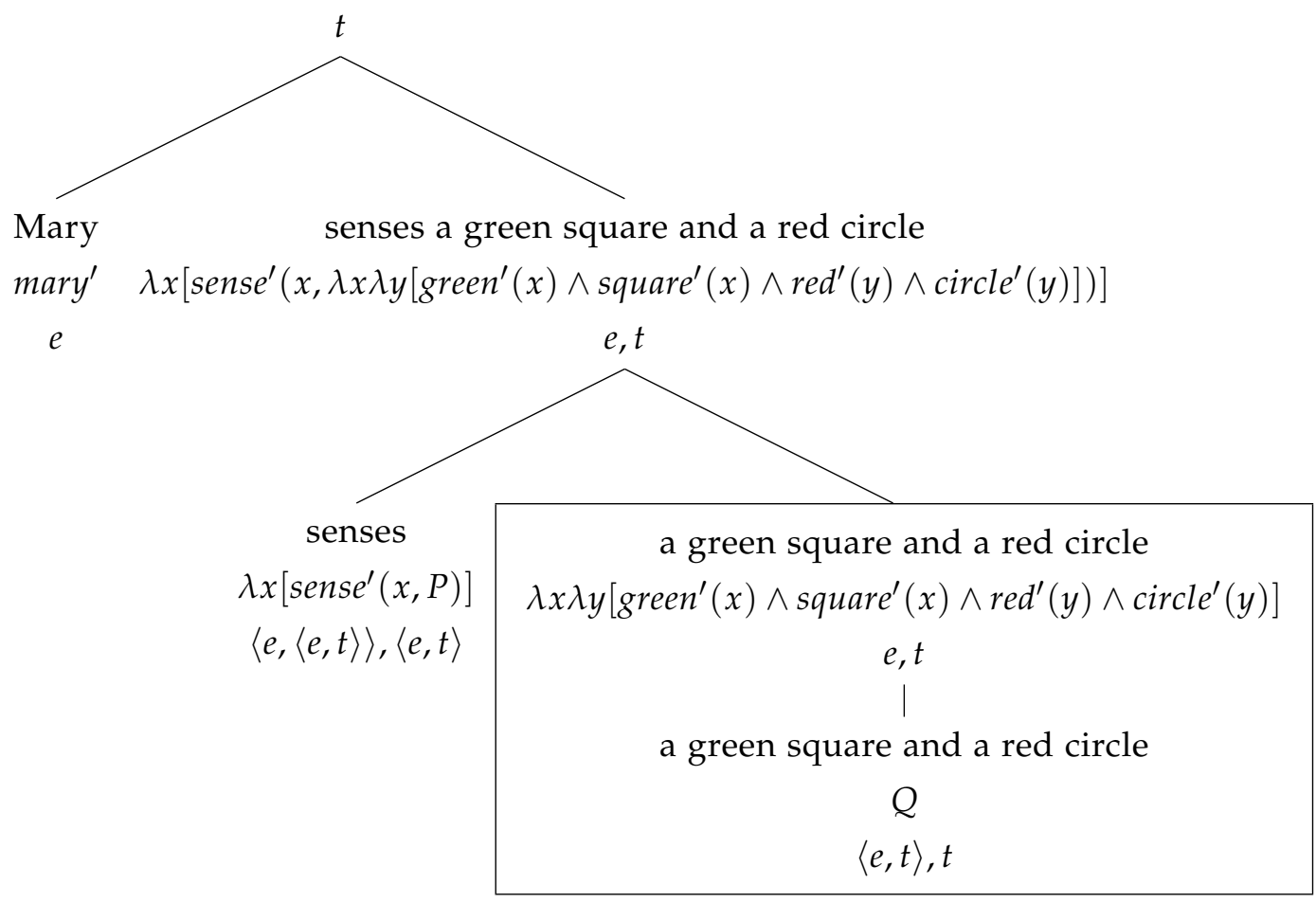

This derivation yields that (28) has the logical form of a relation between Mary and a complex property. Thus, the result matches the logical forms in (11) and (12) which were suggested by Johnston's theory.

With this logical form in hand, we can secure upward monotonicity in the same way we did above: by making use of a lexical analysis. However, the analysis of "seeks" has two distinctive features that must be modified when we consider "senses". ${ }^{25}$ First, (30) makes use of an accessibility relation that holds between worlds whenever one world makes an event in the other successful. Second, it specifies the success of a search in terms of finding. In order for the analogy to hold, we need to specify what the relevant accessibility relation is in the case of sensing, and what the analogue of finding is for states in which we sense properties.

For the case in which we sense visually, I propose the following analogies for

\footnotetext{
${ }^{25}$ Again, nothing turns on my choice of the verb "senses". The exact same approach can be applied to "is perceptually aware of", or any other perceptual verb that can express relations to uninstantiated properties.
} 
the property theorist: finding is to seeking as seeing is to sensing, where seeing is a relation between a perceiving subject and a particular. ${ }^{26}$ Further, success is to finding as accuracy is to sensing. Thus, searches are successful when you find what you seek, and states of sensing are accurate when you see (an instance of) what you sense. ${ }^{27}$ We can then give the following analysis of "senses", where sensing is a relation between a sensing subject and a (complex) property:

Quine + Hintikka Analysis of "sense" (with events)

For any property $P$, individual $x$, world $w$, and time $t$ the following holds: $\operatorname{sense}(P)(x)(w)(t)=1$ iff there is an act of sensing $e$ in $w$ at $t$ of which $x$ is the agent such that the property of being seen by $x$ overlaps the extension of $P$ at $\left(w^{\prime}, t^{\prime}\right)$, for any worlds $w^{\prime}$ and times $t^{\prime}$ such that $(w, t) T\left(w^{\prime}, t^{\prime}\right)$.

In (31), the complex property $P$ serves to specify the accuracy-conditions of an event of sensing. The event of sensing is accurate only if the agent relationally sees particulars that instantiate $P$.

We can see that this analysis validates upward monotonicity by giving the following schematic argument. Suppose that $x$ senses $P$. Any world in which a sensing of $P$ is accurate will be one in which $x$ sees a particular instantiating $P$. But if $P \sqsubseteq Q$, this particular will also instantiate $Q$. Thus one sees a $Q$. It then follows from the right-to-left direction of the biconditional in (31) that $x$ senses $Q$. Therefore, if $x$ senses $P$, then one also senses $Q$. But further, the same reasoning also validates conjunction elimination: if $x$ senses $P$ and $Q$, then $x$ senses $P$.

Returning to our original example, the analysis can be applied as follows. Consider (32):

(32) Mary senses a green square and a red circle.

(32) has the form given in (33):

$$
\text { sense(Mary, } \lambda x \lambda y[\operatorname{green}(x) \wedge \operatorname{square}(x) \wedge \operatorname{red}(y) \wedge \operatorname{circular}(y)])
$$

\footnotetext{
${ }^{26}$ However, there may also be a disanalogy here: findings temporally succeed, or are perhaps the culminations of, searches, whereas seeings do not seem to temporally succeed visual sensings.

${ }^{27}$ Of course, the property representationalist might deny this because it treats relational seeing as fundamental in the analysis of perception. An alternative is to adopt a conjunctive analysis, and claim that sensings are accurate when the subject is appropriately causally related to what she senses. Formally, modifying the analysis in this way is trivial.
} 
We can then apply the analysis above to (33) as follows:

$\operatorname{sense}(\lambda x \lambda y[\operatorname{green}(x) \wedge \operatorname{square}(x) \wedge \operatorname{red}(y) \wedge \operatorname{circular}(y)])(\operatorname{Mary})(w)(t)=1$ iff there is an act of sensing $e$ in $w$ at $t$ of which Mary is the agent such that the property of being seen by Mary overlaps the property $\lambda x \lambda y[$ green $(x)$ $\wedge \operatorname{square}(x) \wedge \operatorname{red}(y) \wedge \operatorname{circular}(y)]$ at $\left(w^{\prime}, t^{\prime}\right)$, for any worlds $w^{\prime}$ and times $t^{\prime}$ such that $(w, t) T\left(w^{\prime}, t^{\prime}\right)$.

Given this application, we can argue as follows. Suppose that Mary senses a green square and a red circle. By the analysis, every world in which Mary's sensation is accurate is one in which she sees a green square and a red circle. But every such world is one in which she sees a green square. Therefore, every world in which her sensation is accurate is one in which she sees a green square. Therefore, by the right-to-left direction of the analysis, Mary senses a green square. Thus, from the fact that Mary senses a green square and a red circle, we can conclude that Mary senses a green square. Similar reasoning allows us to conclude that she senses something green. Thus, by specifying accuracy conditions for Mary's sensation, the analysis in (34) shows how structural relationships between properties yield logical relationships between states of sensing those properties, thus validating upward monotonicity, and solving the inferential problem. ${ }^{28}$

This approach to monotonicity is standard in theorizing about intensional verbs. The semantic value of the complement of an intensional verb is often taken to be an abstract object of some kind (typically a property, generalized quantifier, or proposition), and the truth-conditions of sentences involving such verbs are then specified via the interaction of the verb's modal component with that abstract object. In some cases, such as in the case of propositional attitude verbs, the modal element finds its way into logical form. However, in other cases, such as the case of intensional transitive verbs, it does not. ${ }^{29}$

\footnotetext{
${ }^{28}$ I say that these states are logically related because the analysis serves as an axiom from which the entailment relations between states of sensing can be derived. Here, "logical relations" does not mean relations of structural entailment.

${ }^{29}$ For instance, in von Fintel and Heim |Unpublished, Ch. 2], the modal component of propositional attitude verbs is present in logical form. By contrast, on many accounts of the semantics of intensional transitive verbs, such as Zimmermann [2006], Forbes [2006], and Moltmann [2008], the modal component does not find its way into logical form, but remains at the lexical level.
} 


\section{Solving the Problem for Adverbialism}

The very same strategy can be employed to solve the many-property problem for adverbialism. Solving the problem requires accomplishing two tasks. First, we need to show how adverbial paraphrases can be given a compositional semantics, and then we need to validate upward monotonic inferences.

Consider the adverbial paraphrase of (35), given in (36):

(35) Mary senses a green square and a red circle.

(36) Mary senses (a green square and a red circle)-ly.

Importantly, this adverbial paraphrase is distinct from the paraphrase of (37), given in (38):

(37) Mary senses a red square and a green circle.

(38) Mary senses (a red square and a green circle)-ly.

Given these simple paraphrases, we can think of the semantic value of the objectposition noun phrase as a complex modifier, just as, on the property theorist's view, the complement of "senses" has a complex property as its semantic value. The first step in solving the many-property problem is to show how the semantics of complex modifiers can be derived compositionally. Like on the property view, the complex adverbial modifier can be derived from the ordinary semantic value of "a green square and a red circle" — a generalized quantifier-via a type-shift.

Recall that the ordinary semantic value a noun phrase like "a green square and a red circle" is a generalized quantifier, which, in an extensional setting, is of type $\langle\langle e, t\rangle, t\rangle$. The required type shift takes something of this type and returns a modifier, of type $\langle\langle e, t\rangle,\langle e, t\rangle\rangle$. One way of implementing the shift is to let "-ly" denote a function $f$ that carries out the shift. We can define that function as follows:

(39) $f$ is a function that takes $\lambda P[Q(P)]$ of type $\langle\langle e, t\rangle, t\rangle$ to $\lambda P \lambda x[Q-P(x)]$ of type $\langle\langle e, t\rangle,\langle e, t\rangle\rangle$.

How does this differ from Existential Lowering? The function denoted by "-ly" takes the same input as Existential Lowering-the denotation of a quantified 
$\mathrm{NP}$ - but instead of returning a property that serves as an argument to the verb, it yields a modifier of the verb, denoted by $\lambda P \lambda x[Q-P(x)]$. This modifier takes as an input a verb-of type $\langle e, t\rangle$, and as with modifiers generally, returns something of the same type; in this case, it returns a new, complex verb of type $\langle e, t\rangle$. For example, if our verb is "seeks" and our quantified NP is "a unicorn", the type shift converts the denotation of "a unicorn" into a modifier, which, when combined with the denotation of "seeks", yields the denotation of the complex verb "[a unicorn]-seeks", where [a unicorn]-seeking is a more specific kind of seeking. Moreover, this kind of type-shift is fully general and systematic. Just as with Existential Lowering, the type-shift is defined schematically, and so can be applied to any quantified NP whatsoever. ${ }^{30}$

The hyphenated output of this type-shift may at first seem strange, but it is in fact totally familiar; such hyphenation is used in English to mark a common form of compounding. Consider constructions such as "salmon-fish" or "duckhunt". In these constructions, a nominal is adjoined to the head of the verb with a hyphen, and comes to serve as a modifier that restricts the verb's denotation; salmon-fishing is a particular kind of fishing, and duck-hunting is a particular kind of hunting. ${ }^{31}$ The modifier $\lambda P \lambda x[Q-P(x)]$ functions exactly like these modifiers: it restricts the denotation of the verb to a more specific kind of activity.

Moreover, this is not all that we can say about how the hyphen functions. Just as salmon-fishing is a kind of activity that is successful only if one catches a salmon, and duck-hunting is a kind of activity that is successful only if one catches a duck, [a unicorn]-seeking is a kind of seeking that is successful only if one finds a unicorn-all of these hyphenated constructions denote activities that are directed toward standing in a certain relation to an instance of the kind

$3^{30}$ The type-shift just presented, along with its subsequent explanation using the QuineHintikka analysis, is very similar to the analysis given by Forbes [2006, p. 82]. The basic idea is to type-shift an entire quantified NP to the type of a modifier, and then to explain the behavior of the modifier using an "outcome postulate", which employs the quantified NP in the ordinary way. The fact that the behavior of the modifier is ultimately explained in terms of an ordinary use of the quantified NP guarantees compositionality.

${ }^{31}$ This kind of modification is even more common with noun-phrases: consider, for example, "duck-hunt", "chicken-coop", "three-story building", "one-woman university" and "manysplendoured thing." (The latter three examples come from Forbes 2006, p. 79].) In each of these examples, what was once an argument of a prepositional phrase comes to serve as an attributive adjective that restricts the denotation of the noun. The verbal modifier that is the output of (39) functions exactly analogously, except instead of taking a noun as an input, it takes a verb. Thus, we might call the outputs of our type shift "attributive adverbs." 
that figures into the modifier. This gloss prefigures our ultimate analysis of the modifiers that are the output of the type-shift above. As we will see below, the Quine-Hintikka analysis can be adapted to such modifiers, and can be treated as a meaning postulate governing their behavior. According to the postulate (46) below, what it is to Q-sense is to be the agent of an event with certain successconditions.

With this type-shift in hand, we can provide a compositional semantics for adverbial paraphrases such as (40) by translating each of the lexical items as in (41) and composing them as in (42).

(40) Mary senses (a green square and a red circle)-ly
a. $\quad \operatorname{tr}($ sense $)=\lambda x\left[\right.$ sense $\left.^{\prime}(x)\right]$
b. $\operatorname{tr}($ a green square and a red circle $)=\mathcal{Q}=$ $\lambda P \exists x \exists y\left[\right.$ green $^{\prime}(x) \operatorname{square}^{\prime}(x) \wedge \operatorname{red}^{\prime}(y) \wedge$ circle $\left.^{\prime}(y) \wedge P(x) \wedge P(y)\right]$
c. $\quad \operatorname{tr}($ Mary $)=$ mary
d. $\operatorname{tr}(-1 \mathrm{y})=\mathrm{f}: \lambda P[Q(P)] \Rightarrow \lambda P \lambda x[Q-P(x)]$

(42) Mary senses (a green square and a red circle)-ly

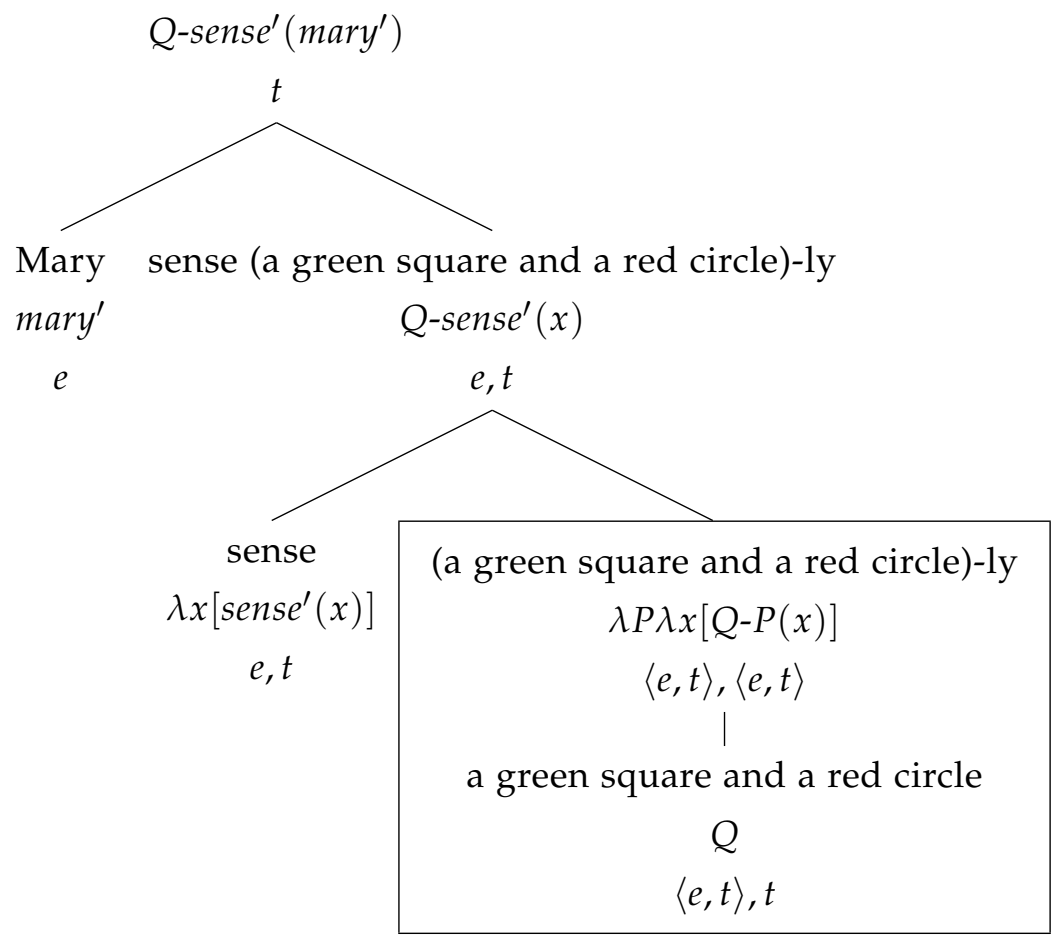


In (42), the boxed portion of the derivation illustrates the application of the type-shifter $f$, whose behavior is specified in (4I-d). The function $f$ can be applied to any generalized quantifier denoted by a noun phrase in the complement of "sense": any such quantifier can be shifted to a modifier using (41-d), and then combined with "sense" as in (42). The result is that to sense $Q$-ly is to $Q$-sense-these two formulations are notational variants. ${ }^{32}$

We now need to show how this compositional adverbial semantics validates upward monotonicity; we need to show that the adverbialist can infer from paraphrases such as (36) to paraphrases like (44) and (45):

(44) Mary senses (something green)-ly.

(45) Mary senses (a square)-ly.

The answer is to again employ the Quine-Hintikka analysis, but to adapt it to adverbial modifiers as follows:

${ }^{32}$ There is another way of deriving the same semantics compositionally without making use of a type-shift. The method is to treat "sense" as semantically incorporating its direct object argument-that is, treat it as on a par with verbs like "salmon-fish" or "duck-hunt". On this method, instead of type-shifting the generalized quantifier that serves as the semantic value of the complement, we can type the incorporating verb so that it has a restrictor argument, as in (43):

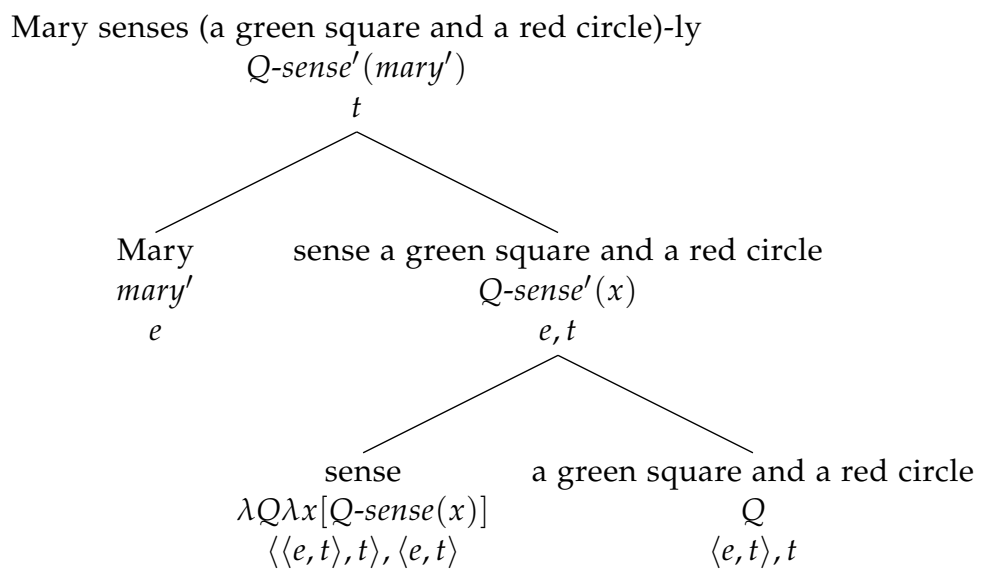

This semantics is very similar to the one given by Dayal [2011, §6.4] for incorporating verbs in Hindi. We can then apply the Quine-Hintikka analysis to the result of this derivation to explain the behavior of the restrictor, and validate upward monotonicity. I have discussed the connection between semantic incorporation and adverbialism in other work (see Author, 2019b). For further discussion of semantic incorporation, see [Carlson, 2006, Dayal, 2011, Borik and Gehrke, 2015. 
Quine + Hintikka Analysis of "sense", adverbial version

For any adverb $Q-l y$, individual $x$, world $w$, and time $t$ the following holds: $Q-l y($ sense $)(x)(w)(t)=1$ iff there is an event of sensing $e$ in $w$ at $t$ of which $x$ is the agent such that the property of being seen by $x$ is an element of $Q$ at $\left(w^{\prime}, t^{\prime}\right)$, for any worlds $w^{\prime}$ and times $t^{\prime}$ such that $(w, t) T$ $\left(w^{\prime}, t^{\prime}\right)$.

On this view, the adverbial modifier $Q$-ly serves to specify the success-conditions for the sensory event. For an event of adverbial sensing to be successful, one must see $Q$, where seeing is a relation between the agent of the event and a particular object, and $Q$ is a quantifier such as "three dogs". Unlike states of sensing on the property view, adverbially modified sensings have no direct objects, and are not relational, but like the property view, the noun-phrases in their object positions contribute type-shifted semantic values to the meanings of sensory ascriptions, and these semantic values specify success-conditions for states of sensing.

We can apply the analysis in (46) to (36) as follows.

(47) (a green square and a red circle)-ly(sense) $($ Mary $)(w)(t)=1$ iff there is an act of sensing $e$ in $w$ at $t$ of which Mary is the agent such that the property of being seen by Mary is an element of $\lambda P[\exists x \exists y[$ green $(x) \wedge$ $\operatorname{square}(x) \wedge \operatorname{red}(y) \wedge \operatorname{circle}(y) \wedge P(x) \wedge P(y)]$ at $\left(w^{\prime}, t^{\prime}\right)$, for any worlds $w^{\prime}$ and times $t^{\prime}$ such that $(w, t) T\left(w^{\prime}, t^{\prime}\right)$.

Given this analysis, we can argue as follows. Suppose that Mary senses (a green square and a red circle)-ly. Given the analysis, every world in which Mary's sensing is successful is one in which she sees a green square and a red circle. But every world in which Mary sees a green square and a red circle is one in which she sees a circle. Thus, every world in which her sensing is successful is one in which she sees a circle. But by the right-to-left direction of the biconditional in (47), it follows that she senses (a circle)-ly. Therefore, if Mary senses (a green square and a red circle)-ly, then she senses (a circle)-ly. Since exactly the same reasoning validates conjunction elimination, adverbial perceptual reports such as (36) and (38) validate upward monotonicity for adverbial modifiers.

The result is a fully compositional semantics for adverbially paraphrased perceptual reports. The semantics solves the expressive problem by forming com- 
plex, quantificational modifiers, and solves the inferential problem by means of the Quine-Hintikka analysis given in (46). This analysis serves as a meaningpostulate governing the behavior of the modifiers deployed in the compositional derivation, and likewise validates upward monotonic inferences. Given the fact that adverbial modifiers are derived in a way exactly analogous to the complex properties above, and governed by an exactly analogous meaning-postulate, the adverbial view is not any more of a post-hoc regimentation of sensory reports than the property view; they are simply different views on the argumentstructure of "senses". Thus, the many-property problem is solved.

\section{Montonicity Inferences: Structural or Non-Structural?}

There are often taken to be two approaches to solving the many-property problemwhat I will call structural approach and the non-structural approach. The structural approach tries to provide adverbial paraphrases with syntactic or logical structure, and then attempts to use this structure to validate the entailments at issue in the inferential problem. ${ }^{33}$ This approach, however, has often been seen as incapable of solving the expressive problem, because it has been thought that building distinct, complex adverbial modifiers requires treating such modifiers as syntactic units.

The non-structural approach, by contrast, accepts this latter conclusion: it treats complex adverbs as syntactic units, and so gives up on validating the inferences at issue using the structural features of sentences. Instead, it tries to validate such inferences by invoking relations of necessitation between states or propositions. Kriegel [2007, 2008, 2011], for example, takes the non-structural approach: he accepts that complex adverbs are syntactic primitives, but claims that some states of sensing entail others on metaphysical grounds: some states of sensing are determinates of others, and so entail them. ${ }^{34}$

33What I here call structural and non-structural approaches go by many names. Evans [1976] calls the structural entailment formal entailment, and contrasts it with semantic entailment. I follow Jackson [2007] in calling the former kind of entailment structural entailment, but while he calls the latter form lexical entailment, I call it non-structural entailment. I use the term "non-structural" because it avoids the implication that such entailments must be semantic. Non-structural entailments are relations of necessitation; they need not be lexical.

${ }^{34}$ There are several different kinds of non-structural entailments that can hold between two states of the world $A$ and $B$. A may a posteriori entail $B$, it may a priori entail $B$, or it may 
Given this distinction, it seems reasonable to ask: is the solution given above a structural solution or a non-structural solution? Are monotonicity inferences structural or non-structural inferences? Before answering this question, it is important to point out that with respect to the content of the solution just given, little turns on the answer we provide; nothing important in the solution above depends on whether the solution is classified as structural or non-structural. We have seen that standard semantic resources solve the many-property problem. The status of these perfectly general resources turns on a range of deep questions in semantics and the philosophy of language such as: What is logical form? What is the relationship between lexical meaning and logical form? Is there a distinction between linguistic knowledge from worldly knowledge? But nothing about the solution above depends on answers to these questions.

However, having said this, we can still go some way toward answering the question of whether monotonicity inferences are structural. First, whether monotonicity inferences count as structural depends on the notion of structure at issue. The monotonicity inferences above are validated by providing a lexical analysis of an intensional verb-in this case "sense". In the analysis, another fully extensional verb that shows up: "see". Thus, the analysis posits a lexical connection between the meanings of "sense" and "see". Such lexical connections are captured using meaning postulates. But often, structural entailments are taken to be entailments that hold independently of meaning postulates linking distinct nonlogical expressions; that is, structural inferences are supposed to be independent of lexical knowledge. Thus, on this definition of what qualifies as a structural inference, monotonicity inferences are not structural.

However, the underlying reasoning that allows us to validate monotonicity inferences may, and often will involve stuctural inferences. For example, to show that "Mary senses a red square" entails that "Mary senses a square", we need to infer from the fact that Mary sees a red square at a world to the fact that she sees a square at that world. This is a structural inference from $\exists x(\operatorname{red}(x) \wedge \operatorname{square}(x)$ $\wedge \operatorname{sees}(\operatorname{Mary}, x))$ to $\exists x(\operatorname{square}(x) \wedge \operatorname{sees}(\operatorname{Mary}, x))$. Thus, if structural inferences are any inferences that involve drawing inferences using first-order logic, then at

analytically entail $B$. Typically, these kinds of entailment are distinguished by the status of the strict conditional $\square A \rightarrow B$. If the conditional is a posteriori, the entailment is a posteriori. If the conditional is a priori, then the entailment is a priori. If the conditional is analytic, then the entailment is analytic. 
least some of the monotonicity inferences validated by "sense" count as structural. Which monotonicity inferences involve structural reasoning will depend on the particular complements involve in the inferences. For example, the analysis above validates inferences like the one from "John senses a dog" to "John senses a mammal", which is not a structural inference. This inference holds in virtue of the (necessary) subset-superset relationship between the extensions of "dog" and "mammal".

We can summarize our conclusions as follows. Monotonicity inferences may count as either structural or non-structural, depending on how we draw the line between structural and non-structural inferences. But what appears to be the case is that, if we rely on an intuitively plausible notion of structure, monotonicity inferences involve both structural and non-structural elements. They first rely on the non-structural, lexical connection between seeing and sensing, but also, in some cases, on the structural relations between properties. These two components, together, solve the inferential problem.

\section{Comparisons}

How does this solution compare to Kriegel's approach to the many-property problem? To use the example we have been discussing, Kriegel's proposal is that sensing (a green circle and a red square)-ly is a determinate of the determinable sensing (a red square)-ly, and so states in which a subject does the former entails states in which she does the latter. The proposal just given is superior to Kriegel's in that it avoids criticisms to which Kriegel's view is subject, and provides a better metaphysical explanation of the relations that hold between states of sensing. To see this, consider the following points.

First, Kriegel's response to the inferential problem relies essentially on the relationship between determinables and determinates. Alex Grzankowski 2018] has criticized Kriegel's view on the grounds that invoking the relationship between determinables and determinates has untoward consequences: it entails that perceiving subjects must, for every state of sensing that is determinable, sense in every way that is a determinate of that determinable, and so be in maximally determinate states of sensing. In the terms used above, Grzankowski shows that relying on determinables and determinates validates downward as 
well as upward monotonicity, and that this leads to absurd consequences.

Second, Kriegel claims that sensing (a green circle and a red square)-ly is a determinate of sensing (a red square)-ly, and so entails it. But he also claims that sensing (rabbit)-ly does not entail sensing (undetached rabbit parts)-ly. But these two claims are in tension: why should an entailment hold between the former two states but not the latter? In the absence of a systematic way of determining which entailments hold between adverbially modified states, claiming that one entailment holds while the other fails seems unprincipled. Kriegel may claim that some states are determinables of others, but he does not provide a justification for doing so.

Third, as we saw above, different verbs have different inferential profiles within their complements; while "sense" validates upward monotonicity, "fears", "lacks", and many other intensional verbs do not. This indicates that entailments between different representational states systematically depend on both the nature of the state in question and the relations between their adverbial modifiers. But Kriegel's proposal ignores how entailments between states systematically depend on these two factors, instead claiming that entailments between states hold only when one unified state is a determinate of another. Thus, Kriegel's proposal misses a crucial explanatory generalization.

The form of adverbialism developed here avoids all of these problems. First, since the proposal just developed does not rely on the relationship between determinables and determinates, and instead employs the Quine-Hintikka analysis, it avoids Grzankowski's criticisms-it validates only upward monotonicity. Second, since the Quine-Hintikka analysis is intensional, it accounts for the intensionality within the complement of "sense", and provides a principled way of distinguishing between adverbially modified states up to necessary equivalence of their modifiers. ${ }^{35}$ Third, this proposal provides a systematic account of the

35 In distinguishing between states of sensing only up to necessary equivalence of adverbial modifiers, this form of adverbialism is not hyperintensional. Accordingly, if a subject senses $P$-ly, and necessarily, all and only $P$ s are $Q$ s, then the subject senses $Q$-ly. Further, it follows from the Quine-Hintikka analysis that if a subject senses anything, the subject senses the property of being self-identical (or any other property that everything has necessarily). These consequences are features of a possible-worlds approach to spelling out success-conditions, and are exactly analogous to the problems occasioned by the Hintikka analysis of propositional attitudes. However, there are well-known ways of addressing these problems. For one approach to hyperintensionality, see Forbes [2006. Ch. 8]. For an attempt to solve the analogue of the logical omniscience problem, see Zimmermann 2006. 
entailment relations between states of sensing that reveals how they depend on both the nature of the state in question and the relations between modifiers of that state. Given a verb $V$ and two adverbs $Q$-ly and $R$-ly, it determines whether $x V \mathrm{~s} Q$-ly entails that $x V \mathrm{~s} R$-ly in terms of whether fulfillment of the successconditions specified by $Q$-ly likewise specify the success-conditions specified by $R-1 y$.

The present proposal also has the advantage of being fully compositional. Kriegel himself does not discuss the question of compositionality, but his solution appears to be non-compositional: determinate to determinable inferences rely on our knowledge of the relations between the modifiers denoted by syntactically simple expressions. But given that such modifiers can be arbitrarily complex, it appears that even if certain adverbially modified states of sensing necessitate each other, we could not come to know that they do. ${ }^{36}$ By contrast, while the view developed here does require some knowledge of relations between lexical items-between the meanings of "sense" and "see"—it is fully compositional, and can thus underwrite our knowledge of entailments between states of sensing that are modified in arbitrarily complex ways.

Finally, the solution presented here is based on a mechanism that is totally standard in semantics, and is necessary for purposes other than solving adverbialism's inferential problem. Adopting it subsumes the inferential problem for adverbialism — which has at times appeared intractable — under a much broader semantic problem that is not intractable. On the contrary, it is a problem for which there is a widely accepted solution. As such, there appears to be no reason why those inclined towards Kriegel's adverbialism—or toward other forms of adverbialism—should resist adopting it.

\section{Conclusion}

The foregoing arguments show that with respect to the many-property problem, a range of property views in the philosophy of perception are on a par with adverbialism. Both property views and adverbial views treat the complements of perceptual verbs in nonstandard ways. Property theorists treat them as denoting

\footnotetext{
${ }^{36}$ This point has been noted already by Alexander Dinges 2015, p. 233, n. 2]; here I simply reiterate it. I am grateful to an anonymous referee for Philosophical Studies for rightly pointing this out.
} 
complex properties, while the adverbialist treats them as denoting complex adverbial modifiers. Further, both of these nonstandard treatments are derived from the ordinary meaning of the direct-object noun phrase via a type-shift. Both views can then make use of the Quine-Hintikka analysis-which shows how the structural relationships between properties determine relationships of entailment between states of sensing - to validate upward monotonic inferences.

The resulting form of adverbialism differs from traditional adverbial views in that it involves success-conditions, and these success-conditions are essential to the adverbialist's ability to overcome the many-property problem. Insofar as this form of adverbialism involves success-conditions, and specifies these successconditions in terms of relational seeings, it takes relational seeing as explanatorily fundamental. However, the view is one on which the success-conditions of adverbial perceptual states are specified by adverbial modifiers, and on which in the case of a hallucination, there simply is no object to which the hallucinating subject is related. This captures the main desiderata on an adverbial view of perception.

The result is that views in the philosophy of perception that invoke relations to uninstantiated properties are not as different from adverbialism as many have thought. The main difference between them is that property views typically maintain that the direct object of hallucinatory experiences is a property, while the adverbialist maintains that hallucinations have no direct objects. Thus, their analysis of the structure of perceptual states differ. However, while the adverbialist and the representationalist differ concerning the structure of perceptual states, both take states to involve the specifications of conditions-accuracy conditions for the representationalist, and success-conditions for the adverbialist. Insofar as both adverbialism and representationalism involve the specification of such conditions for perceptual states, and spell them out using the Quine-Hintikka analysis, they have much much more in common than is typically thought.

\section{References}

Travis Banick. How to be an adverbialist about phenomenal intentionality. Synthese, forthcoming.

George Bealer. Quality and Concept. Clarendon Press, Oxford, 1982. 
Olga Borik and Berit Gehrke, editors. The Syntax and Semantics of PseudoIncorporation. Syntax and Semantics. Brill, Leiden and Boston, 2015.

David Bourget. Implications of intensional perceptual ascriptions for relationalism, disjunctivism, and representationalism about perceptual experience. Erkenntnis, 2017.

David Bourget. Relational vs adverbial conceptions of phenomenal intentionality. In A. Sullivan, editor, Sensations, Thoughts, and Language: Essays in honor of Brian Loar. Routledge, forthcoming.

Greg Carlson. The meaningful bounds of incorporation. In Svetlana Vogeleer and Liliane Tasmowski, editors, Non-definiteness and plurality, Linguistics Today 95. John Benjamins Publishing Company, 2006.

Roderick Chisholm. Perceiving: A philosophical study. In David Rosenthal, editor, The Nature of Mind, chapter 11. Oxford University Press, 1956.

Justin D'Ambrosio. A new perceptual adverbialism. Journal of Philosophy, 116(8): 413-446, 2019.

Veneeta Dayal. Hindi pseudo-incorporation. Natural Language and Linguistic Theory, 29(1):123-167, 2011.

Alexander Dinges. The many-relations problem for adverbialism. Analysis, 75 (2):231-237, 2015.

Fred Dretske. Naturalizing the Mind. The Jean Nicod Lectures. The MIT Press, 1995.

Fred Dretske. The mind's awareness of itself. Philosophical Studies, 95:103-124, 1999.

Fred Dretske. Experience as representation. Philosophical Issues, 13(1):67-82, 2003.

C.J. Ducasse. Moore's refutation of idealism. In P. Schilpp, editor, The Philosophy of G.E. Moore, pages 223-252. Northwestern University Press, Chicago, 1942.

Gareth Evans. Semantic structure and logical form. In Collected Papers. Clarendon Press, Oxford, 1976.

Graeme Forbes. Attitude Problems. Oxford University Press, Oxford, 2006.

Peter Forrest. Universals as sense-data. Philosophy and Phenomenological Research, 71:622-631, 2005.

John Foster. The Nature of Perception. Oxford University Press, Oxford, 2000.

Alex Grzankowski. The determinable-determinate distinction can't save adverbialism. Analysis, 78(1):45-52, 2018.

Herman Hendriks. Studied Flexibility. PhD thesis, University of Amsterdam, Amsterdam, 1993.

Jaako Hintikka. Knowledge and Belief: An Introduction to the Logic of the Two Notions. Cornell University Press, 1962.

Brendan Balcerak Jackson. Beyond logical form. Philosophical Studies, 132(2), January 2007. 
Frank Jackson. On the adverbial analysis of visual experience. Metaphilosophy, 6 (2):127-135, April 1975.

Mark Johnston. The obscure object of hallucination. Philosophical Studies, 120 (1-3):113-183, 2004.

Uriah Kriegel. Intensional inexistence and phenomenal intentionality. Philosophical Perspectives, 21(Philosophy of Mind):307-340, 2007.

Uriah Kriegel. The dispensability of (merely) intentional objects. Philosophical Studies, 141:79-95, August 2008.

Uriah Kriegel. The veil of abstracta. Philosophical Issues, 21(The Epistemology of Perception):245-267, 2011.

Angela Mendelovici. The Phenomenal Basis of Intentionality. Oxford University Press, New York, 2018.

Friederike Moltmann. Intensional verbs and their intentional objects. Natural Language Semantics, 16:239-270, 2008.

Adam Pautz. Intentionalism and perceptual presence. Philosophical Perspectives, 21:495-541, 2007.

Adam Pautz. Why explain visual experience in terms of content? In Bence Nanay, editor, Perceiving the World, pages 254-309. New York, 2010.

Aynat Rubinstein. Straddling the line between attitude verbs and necessity modals. In Andrés Salanova Ana Arregui, María Luisa Rivero, editor, Modality Across Syntactic Categories, chapter 7. Oxford University Press, Oxford and New York, 2017.

R. M. Sainsbury. Thinking about Things. Oxford University Press, Oxford, 2018.

Susanna Schellenberg. Externalism and the gappy content of hallucination. In Fiona Macpherson and Dimitris Platchias, editors, Hallucination: Philosophy and Psychology. MIT Press, Cambridge, MA, 2013.

Susanna Schellenberg. The relational and representational character of perceptual experience. In Berit Brogaard, editor, Does Perception Have Content?, pages 199-219. Oxford University Press, New York and Oxford, 2014.

Wilfrid Sellars. The adverbial theory of the objects of sensation. Metaphilosophy, 6(2):144-160, 1975 .

Michael Tye. The adverbial theory: A defence of sellars against jackson. Metaphilosophy, 6(2):136-143, 1975.

Michael Tye. The adverbial aproach to visual experience. The Philosophical Review, 93(2):195-225, 1984.

Michael Tye. The Metaphysics of Mind. Cambridge University Press, Cambridge, 1989.

Michael Tye. Consciousness Revisited: Materialism without Phenomenal Concepts. Representation and Mind. MIT Press, Cambridge and London, 2009.

Michael Tye. What is the content of a hallucinatory experience? In Berit Brogaard, editor, Does Perception Have Content?, chapter 10. Oxford, Blackwell, Oxford 
and New York, December 2014a.

Michael Tye. Transparency, qualia realism and representationalism. Philosophical Studies, 170:39-57, 2014b.

Johan van Benthem. Questions about quantifiers. Journal of Symbolic Logic, 49(2): 443-466, June 1984.

Kai von Fintel and Irene Heim. Intensional Semantics. Lecture Notes, Cambridge, MA, Unpublished.

Casey Woodling. The limits of adverbialism about intentionality. Inquiry, 59(5): 488-512, February 2016.

Thomas Ede Zimmermann. Monotonicity in opaque verbs. Linguistics and Philosophy, 29:715-761, December 2006. 